\title{
Impact of Drug Delivery Systems on Neuraxial Labor Analgesia
}

\author{
Srividhya Jayant Iyer $\cdot$ Alex T. H. Sia
}

Published online: 11 August 2013

C Springer Science + Business Media New York 2013

\begin{abstract}
Neuraxial analgesia for the treatment of labor pain has proven to be efficacious and safe over the past few decades. At present, several new strategies are being developed to further enhance pain relief and maternal satisfaction of our parturients while providing them with greater autonomy over their own analgesic regimen. The recent years have witnessed the development of new infusion pumps that are more interactive, flexible and allow titration of analgesia according to an individual parturient's need for pain relief as labor progresses. In this review, we examine the several strategies for maintenance of neuraxial labor analgesia with a focus on the impact of drug delivery systems in the past, present and future.
\end{abstract}

Keywords Pump · Technology $\cdot$ Advances $\cdot$ Labor · Analgesia

\section{Introduction}

Labor pain is arguably one of the most painful experiences a woman can undergo [1]. Although invasive, epidural analgesia is considered safe and is proven to provide the most efficacious pain relief in contemporary practice [2].

Labor pain is influenced by several factors, which include duration and progression of labor, presence of dysfunctional

S. J. Iyer $(\bowtie) \cdot$ A. T. H. Sia

Department of Women's Anaesthesia, KK Women's and

Children's Hospital, 100 Bukit Timah Road, Singapore 229899,

Singapore

e-mail: vidhyamed@gmail.com labor and use of oxytocin augmentation regimens [3]. Cultural, social and psychological beliefs may also influence the way pain is perceived by the laboring patient $[3,4]$. It is important to recognize that analgesic requirements change as labor progresses [3]. The multi-factorial and dynamic nature of labor pain explains the inter-patient variability in analgesic requirements. It also highlights the importance of tailoring a suitable epidural analgesic regimen to meet the needs of every individual parturient so as to enable mothers to have a satisfying birth experience.

Extensive research over the past two decades has been dedicated to further enhancing the safety and efficacy of neuraxial labor analgesia [5]. Efforts are aimed at improving maternal satisfaction while minimizing episodes of breakthrough pain, local anesthetic consumption and unwanted side effects such as motor block. This has lead to the development of patient controlled epidural analgesia (PCEA), the debate on continuous epidural infusion (CEI) versus intermittent mandatory boluses, as well as developments such as computer-integrated PCEA (CIPCEA) that automatically adjusts background infusion rates based on an individual parturient's need for analgesia as labor progresses [4, 6].

Pump technology has always played a pivotal role in the delivery of epidural local anesthetic in the maintenance of neuraxial labor analgesia. Advances in pump technology are being achieved by integration of computer systems into analgesic modalities [5] to create 'smart pumps' that are better suited to providing a tailored analgesic regimen for optimal pain relief and greater maternal satisfaction.

Maintenance of labor epidural analgesia is rapidly evolving in the field of obstetric anesthesia. This review aims to examine this topic with a focus on the impact of drug delivery systems on neuraxial labor analgesia in the past, present and future. 


\section{The Past}

Review of literature from several decades ago reveal that a single bolus injection of local anesthetic solution to achieve a lumbar epidural or caudal block was used as routine forms of neuraxial analgesia in labor [7]. This procedure was typically performed when the parturient was welladvanced in labor and delivery was imminent. The analgesia provided from this technique was often insufficient and at best able to only provide temporary relief.

Hence, the practice of placing an indwelling plastic catheter in the epidural space was undertaken to allow repeated boluses of local anesthetic for earlier and more satisfactory pain relief in labor [7]. These manual boluses were given as "top-ups" by the clinician when labor pain returned or worsened. With greater flexibility compared to the single injection method, Bromage [8] reported this as a safe and satisfactory technique while it was considered by Moore [9] to be "the ultimate in providing pain relief to the parturient".

However, the disadvantages of these intermittent, irregular 'top-ups' for the maintenance of labor analgesia were soon recognized, the most significant of these was the waning of analgesic effect and the return of pain before the next dose of local anesthetic [10]. Other concerns included repeated injections causing sudden increase in sympathetic blockade, increased risk of a total spinal or local anesthetic toxicity [11]. The idea for continuous infusion of local anesthetic through the catheter (at a fixed or pre-set rate to be changed only by the clinician) which came to be known as CEI subsequently took root. This technique was thought to prevent pain recurrence as there was no waiting for analgesic effect to wear off before administering additional local anesthetic. It was also believed to be safer with less likelihood of accidental overdose.

Several devices were used to achieve CEI worldwide. Three decades ago, Davies and Fettes [11] described a method for continuous infusion epidural anesthesia using a $6 \mathrm{ml} / \mathrm{h}$ capillary infusion device (Intraflo CFS-06F) instead of traditional infusion pumps which were expensive and bulky. With time, infusion pumps were improvised and became more affordable. CEI, with the use of simple infusion pump devices that allow manual adjustment of infusion rate, became the technique of choice for the maintenance of neuraxial analgesia in labor in many countries and continues to remain so to date.

Although pain relief obtained with CEI was superior to that of the manual 'top-up' method, breakthrough pain requiring "rescue" boluses by the anesthetist still continued to occur. Higher infusion rates decreased the need for rescue boluses but resulted in greater incidence of motor block in parturients $[10,12,13]$. This occurred despite the use of low-dose epidural infusion in response to the findings of the landmark COMET trial conducted in the United Kingdom [14]. This trial demonstrated that there were higher rates of normal vaginal delivery (with reduction in instrumental vaginal delivery) in the low-dose ( $0.1 \%$ bupivacaine and $2 \mathrm{mcg} / \mathrm{ml}$ fentanyl) maintenance group compared with those who received the traditional, higher epidural concentration $(0.25 \%$ bupivacaine).

With persisting problems of breakthrough pain and maternal motor block, it was only logical to continue in the quest for alternative modes of drug delivery that promised greater maternal satisfaction and superior results. This was to be achieved with the promising advances in pump technology that were made in the last two decades.

\section{The Present}

\section{Patient-Controlled Epidural Analgesia (PCEA)}

Introduced more than two decades ago, PCEA allows parturients to self-administer intermittent boluses of epidural solution. This technique enables them to tailor their analgesic requirements to their level of pain, which may not only vary from one patient to another, but also as labor progresses or when labor augmentation regimens are employed [3].

PCEA has proven to be a safe and effective mode of drug delivery and there is no evidence of any adverse impact on obstetric and neonatal outcomes compared with a continuous epidural infusion [15]. The invention of PCEA has provided autonomy and flexibility of the analgesic regimen for each individual parturient. Benefits of PCEA include: decreased consumption of local anesthetic without compromising quality of analgesia, reduced incidence of motor block of lower extremities, decreased physician workload due to a reduction of unscheduled clinician interventions and overall improved maternal satisfaction $[3,5,16]$.

The earliest study on PCEA demonstrating its advantages over CEI was by Gambling et al. [17]. Their team used a PCA (Patient-controlled analgesia) pump that was used routinely for providing intravenous opioid analgesia. The PCA pump had a custom-made control device that allowed a continuous infusion of local anesthetic, with the ability to give additional incremental boluses by activation of a patient demand button. The rate of basal infusion, incremental dose and lock out period were all programmable. Since then, studies on PCEA and usage of PCEA on parturients continued to be done with pumps that are used for PCA intravenous analgesia [18]. At our institution, the Graseby 9300 PCA pump was used for this purpose [1922] for a number of years. Currently, many institutions worldwide that have evolved from CEI to PCEA as the 
routine mode of maintenance of neuraxial labor analgesia have done so using pumps that are similar to those used for PCA (intravenous analgesia).

Over the past decade, PCEA techniques have been further refined to include finding the ideal settings for basal infusion rate, bolus-volume and lock-out interval [5, 16]. This, together with the advent of other modalities such as automated intermittent mandatory boluses (AMB), computer-integrated PCEA (CIPCEA), as well as the integration of PCEA in these modalities have all led to the development of 'smarter' pump modalities. Technology has allowed integration of computer systems into syringe pumps that are now better suited to tailoring an individualized analgesic regimen for the parturient.

\section{Automated Intermittent Mandatory Boluses (AMB)}

Another major development in the field of obstetric anesthesia in the recent years is the automated intermittent mandatory boluses (AMB) modality, also known as programmed intermittent epidural boluses (PIEB). As the name implies, this technology serves to administer the same total hourly amount of local anesthetic as intermittent boluses rather than as a continuous infusion (e.g., a $5 \mathrm{ml}$ bolus is administered over a few minutes every hour instead of a $5 \mathrm{ml}$ infusion over $1 \mathrm{~h}$ ).

Many studies have shown AMB to be more effective than CEI for labor analgesia [3, 5]. Some of the reported advantages include reduced need for epidural rescue medication, lower epidural drug use, longer time to first rescue bolus for breakthrough pain, longer duration of analgesia after combined spinal epidural (CSE) and superior or equivalent analgesia as compared to the continuous infusion group [23, 24].

There are several potential reasons for these findings. It has been shown that the spread of the infusate from a multiorifice catheter is more extensive and uniform when intermittent boluses are used compared to a continuous infusion, possibly related to the higher injectate pressure generated during a bolus injection [25, 26]. Also, a continuous infusion was shown to result in flow through the proximal hole of a multi-orifice catheter, only, whereas boluses resulted in the solution exiting the catheter via all the holes [25]. Lastly, it is prescribed that when AMB is used with a CSE technique, the high driving pressure generated when administering a bolus may allow some degree of direct transfer of the local anesthetic solution into the intrathecal space via the dural hole, resulting in an improved quality of the resulting analgesia [23].

Chua and Sia [23] compared the efficacy of intermittent boluses (AMB) against CEI in 42 nulliparous parturients after successful induction of CSE analgesia. The Graseby 9200 pump was used for administering boluses of $5 \mathrm{ml}$ every hour in the AMB group while the Terumo infusion syringe pump was used in the CEI group to run a continuous infusion of local anesthetic at $5 \mathrm{ml} / \mathrm{h}$. The results showed that patients in the AMB group had better pain scores and longer duration of analgesia after CSE before the first episode of breakthrough pain. Lim et al. [27] subsequently postulated that the use of small or low volume bolus may have a better safety profile and investigated if the benefits previously demonstrated with AMB were still retained if a low bolus volume were to be used. Fifty parturients were randomized to either AMB group $(2.5 \mathrm{ml}$ every $15 \mathrm{~min}$ via a rhythmic pump) or CEI group $(10 \mathrm{ml} / \mathrm{h}$ via Terumo infusion syringe pump). There was no significant difference between the two groups in terms of breakthrough pain, indicative that low-volume boluses did not confer any improvement of analgesic efficacy when compared to CEI.

Soon, interest in the integration of PCEA with AMB grew, with the aim to further improve analgesic outcome and patient satisfaction. At our institution, we integrated PCEA with AMB in a one-pump system and studied 42 parturients in early labor who were randomized to receive either a PCEA with continuous epidural infusion (PCEA and CEI at $5 \mathrm{ml} / \mathrm{h}$ ) or a PCEA with AMB (PCEA and AMB of $5 \mathrm{ml}$ every hour) [28]. To do so, we devised a computer program that enables an ordinary continuous infusion pump to function as a PCEA pump, with the additional ability to deliver mandatory intermittent boluses. These mandatory boluses were given in addition to the demand-driven PCEA boluses by the patient. The PCEA + AMB and PCEA + CEI program source codes were compiled in the Borland Pascal language on a Windows Operating Systems. The infusion pump, IVAC P700 (Alaris, UK) was connected via RS232 serial ports to a lap-top (IBM Thinkpad) (which contained the software for different PCEA algorithms) to establish two-way communication between the lap-top and infusion pump (Fig. 1). A remote control was given to the parturient which she was asked to activate when she required a PCEA bolus. This set-up functioned well and the results demonstrated that the PCEA and AMB group had reduced overall consumption of ropivacaine along with longer duration of analgesia before the first PCEA selfbolus.

In a subsequent study, we further improvised by creating a less cumbersome and more portable epidural drug delivery system [29]. Leo et al., randomized 62 laboring patients to receive either PCEA and AMB or PCEA and CEI. This time, a modified Perfusor Compact $\mathrm{S}$ infusion pump (B. Braun, Germany) was connected to a personal digital assistant (PDA), Dell Axim X51v (Dell, USA) operating Windows Mobile version 5 software (Microsoft, USA) (Fig. 2). Again, program source codes for both regimens were loaded into the PDA and both programs 


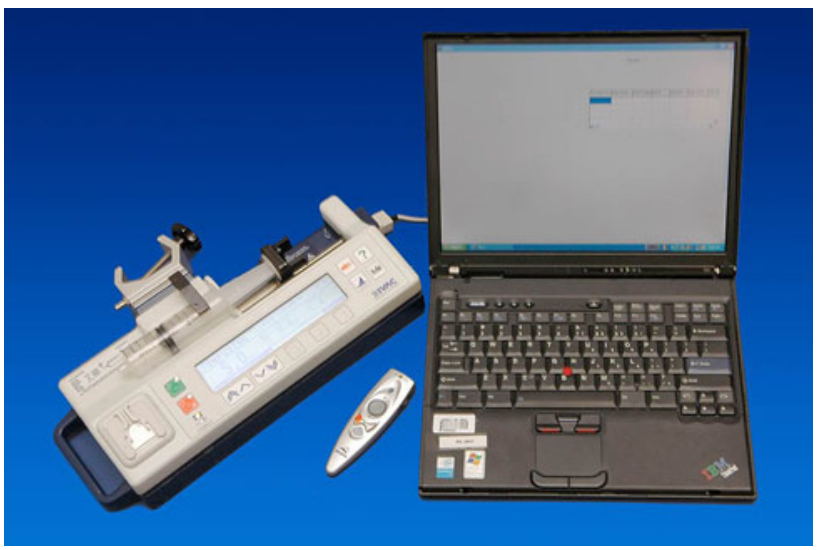

Fig. 1 First generation computer integrated infusion pump set-up using an IBM Thinkpad laptop (IBM, USA) connected to a modified infusion pump (IVAC P700, Alaris, UK)

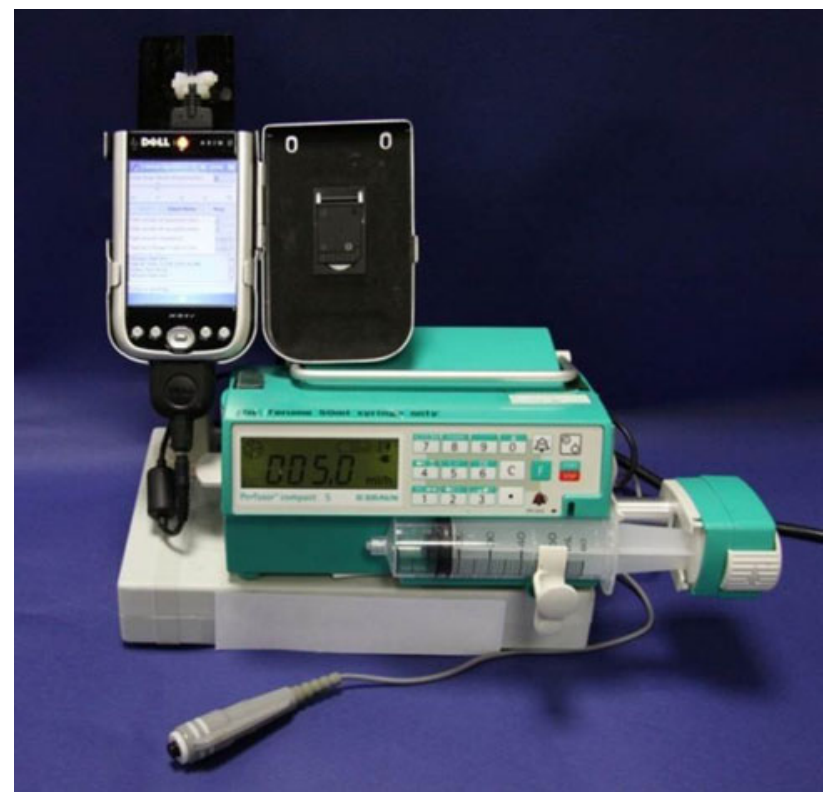

Fig. 2 Portable epidural drug delivery system driven by a personal digital assistant (PDA) operating on Windows Mobile systems software and connected to a modified Compact $\mathrm{S}$ infusion pump (B.Braun)

underwent rigorous in vitro testing at our Biomedical Engineering Unit and by all investigators independently before being applied to patients in a clinical setting [29]. The results of this study were similar to those of our previous study, with greater patient satisfaction in the PCEA and AMB group.

Our results confirm those of other studies that have compared AMB (PIEB) with CEI in combination with a PCEA with the use of a dual pump system: one pump administering either AMB or CEI for the maintenance of analgesia, while the second pump is used to administer PCEA to treat breakthrough pain [30-32]. Epidural infusion tubing from both pumps are then connected via a three-way stopcock to the hub of the epidural catheter. Wong et al. [30] analyzed 126 parturients using this dual set up and found that the AMB and PCEA group enjoyed similar analgesia, but with a smaller bupivacaine dose and better patient satisfaction compared with the CEI and PCEA group for maintenance of epidural labor analgesia.

Capogna et al. [32] studied the effects of AMB and PCEA versus CEI and PCEA on maternal motor function and labor outcome on 145 nulliparous patients. Their results demonstrated that the incidence of maternal motor block and instrumental delivery was significantly lower in the AMB group compared to the CEI group. These investigators also used two pumps (Gemstar, USA), equipped with anti-reflux epidural infusion tubing and three-way stop cock connecting tubing from each pump to the patient's epidural catheter. In this study, solutions of different strengths were used in the two pumps $(0.125 \%$ levobupivacaine for PCEA pump and $0.0625 \%$ levobupivacaine with sufentanil $0.5 \mathrm{mcg} / \mathrm{ml}$ for the AMB and CEI pump). Capogna and Stirparo [10] had previously noted that a high rate of breakthrough pain requiring clinician intervention was recorded in studies using the same concentration of local anesthetic for both PCEA and AMB boluses. While assembling a dual pump set-up is technically easier than integrating computer systems into infusion pumps, the lack of communication between PCEA and the AMB (or CEI) modes in a dual pump set-up may risk a large volume bolus being delivered to the patient if the PCEA bolus and AMB are activated at the same time.

A recent systematic review and meta-analysis by George et al. [33•], comparing CEI to AMB, with or without PCEA, included nine randomized controlled trials analyzing a total of 344 patients who received CEI and 350 who received AMB. The results showed reduced local anesthetic consumption, shorter second stage of labor and higher maternal satisfaction in the AMB group. However, as there were no significant differences in outcomes such as mode of delivery and required anesthetic interventions, the authors concluded that more research is needed to confirm the superiority of the AMB/PCEA compared with CEI/ PCEA and to determine the ideal AMB/PCEA regimen to further improve labor analgesia and have a favorable effect on obstetric outcomes. They asserted that these improved outcomes would then justify the added costs of developing and implementing the new pump technology required for this technique.

\section{Computer-Integrated Patient Controlled Epidural Analgesia}

Despite allowing the parturient to titrate their epidural analgesia via self administered boluses, a conventional 
PCEA pump does not have the ability to provide a varying basal rate without clinician intervention. Even though a basal infusion may not be required in early labor, its role is likely to become important as pain escalates with the progress of labor or when labor is augmented. Thus, having a drug delivery system which is responsive to the patient's needs may provide more efficacious analgesia.

In our earlier studies, we had devised a program, based on a novel clinical algorithm, which converted an ordinary infusion pump into a computer-integrated (CI)-PCEA system that is able to automatically adjust the basal infusion rate, based on the number of PCEA demands by the patient over the last hour. This interactive delivery system constantly records the history of the patient's analgesic requirement and adjusts the basal in $5 \mathrm{cc}$ increments if the patient requires one, two or three demand boluses, respectively, in the past hour. If there are no demands for the previous hour, the pump decreases the basal infusion rate by increments of $5 \mathrm{ml} / \mathrm{h}$.

This first generation CIPCEA was made possible by accomplishing two-way communication between a IVAC P700 infusion pump and an IBM Thinkpad lap-top computer as described above (Fig. 1). Each parturient was also given a remote controlled (wireless) modified hand-held computer "mouse" analagous to a PCEA button which served as the interface between the patient and the CIPCEA/infusion pump set [34]. Our pilot study that utilized this set-up compared CEI of $10 \mathrm{ml} / \mathrm{h}$ versus CIPCEA regimen on 40 parturients who were randomized after induction of CSE analgesia [34]. Our results showed a significant reduction in the incidence of breakthrough pain in the CIPCEA group, without an increase in the local anesthetic consumption or incidence of side effects.

Our subsequent studies compared CIPCEA versus PCEA regimens with or without basal infusion $[6,35]$. We also used the improvised and more portable Compact $\mathrm{S}$ infusion pump (B. Braun) connected to a PDA (Fig. 2) for our study by Sng et al. [6]. The results showed greater maternal satisfaction in the CIPCEA group without any difference in time-weighted consumption of local anesthetic between the two groups. However, we could not demonstrate any statistically significant difference in the incidence of breakthrough pain between the two groups in these studies.

In our most recent study, we integrated the concept of AMB and CIPCEA to develop a method of administering variable-frequency automated boluses at a rate proportional to the patient's needs [36••]. In this new concept, while automated boluses of $5 \mathrm{ml}$ were given in addition to patient-controlled boluses, the frequency of the automated boluses (AMB) was dependent on the history of patient's analgesic requirement over the past hour. If the patient requires one, two, three or four demand boluses in the past hour, the AMB frequency would be adjusted to every 60 , 30,20 or $15 \mathrm{~min}$, respectively. If there were no demands for the previous hour, then the AMB frequency would decrease in a step-wise fashion. We found that when compared with conventional PCEA, the variable frequency AMB reported lower incidence of breakthrough pain as well as higher maternal satisfaction scores. There were no differences in the time weighted hourly consumption of ropivacaine, maternal side effects or obstetric and neonatal outcomes.

In the time period that we have been using PCEA and its derivatives (CIPCEA, AMB/PCEA and etc.) in all our laboring patients, we have noticed an improvement in terms of reduced episodes of breakthrough pain requiring anesthetist attendance, reduced workload, and increased maternal satisfaction. Our newest epidural drug delivery system consists of a Tablet PC (Hewlett Packard Compaq 2710p) operating on Microsoft windows XP Tablet PC Edition 2005 (Microsoft, USA) connected to a modified Perfusor Compact S infusion pump (B. Braun) (Fig. 3). Two-way communication between the pump and HP Tablet PC is accomplished by connecting the pump serial ports to the USB port on the Tablet PC. The program source codes for the different regimens being used are contained within the Tablet PC. Each room in the delivery suite is equipped with this WIFI enabled set-up, which has proven to be equally compact, yet more user friendly than our previous PDA set-up. All the Tablet PCs and pumps in our 32-bed delivery suite are linked via a WIFI network which allows real-time data capture and to remote monitoring of all the pumps from a central monitoring system.

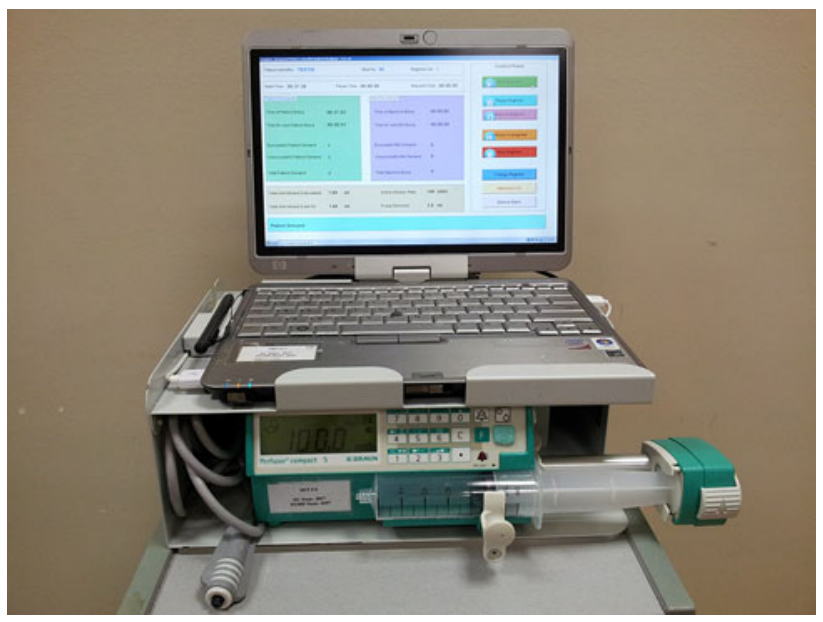

Fig. 3 The patient-controlled epidural analgesia system utilizing a Hewlett Packard Compaq Tablet PC connected to a modified B. Braun Perfusor R Compact S infusion pump 


\section{The Future}

Since the beginning of the new millenium, the benefits of using various regimens such as PCEA, AMB and CIPCEA over CEI in proving labor neuraxial analgesia are being increasingly recognized. However, there is a continuing need to further define and refine, to provide even better analgesia, to increase maternal satisfaction, and to improve obstetric outcomes. The focus going forward is on utilizing new technology to integrate the above described modalities in order to further improve efficacy of labor epidural analgesia in a cost effective manner. The availability of such interactive and remotely monitored pumps will be an exciting development that we look forward to in the future.

\section{Conclusions}

Positive findings from several trials have helped to establish different modalities of maintenance of epidural labor analgesia such as PCEA, AMB and CIPCEA. This has been made possible by advances in technology that have provided us with superior drug delivery systems. It is important for clinicians to recognize the multi-factorial, dynamic and individual nature of labor pain as we continue to utilize technological advances to customize an appropriate regimen for each of our parturients. In this way, we can provide a safe, pleasant and memorable birthing experience for them.

\section{Compliance with Ethics Guidelines}

Conflict of Interest Srividhya Jayant Iyer declares that she has no conflict of interest. Alex T. H. Sia is supported by a Grant from the Office of the Prime Minister.

Human and Animal Rights and Informed Consent This article does not contain any studies with human or animal subjects performed by any of the authors.

\section{References}

Papers of particular interest, published recently, have been highlighted as:

- Of importance

-• Of major importance

1. Melzack R. The myth of painless childbirth (the John J. Bonica lecture). Pain. 1984;19(4):321-37.

2. Mhyre JM. What's new in obstetric anesthesia? Int J Obstet Anesth. 2011;20(2):149-59.

3. Leo S, Sia AT. Maintaining labour epidural analgesia: what is the best option? Curr Opin Anaesthesiol. 2008;21(3):263-9.

4. Srividhya Jayant Iyer SL. Maintenance of neuraxial block in labor analgesia. Trends Anaesth Crit Care. 2012;2(4):199-204.
5. Loubert C, Hinova A, Fernando R. Update on modern neuraxial analgesia in labour: a review of the literature of the last 5 years. Anaesthesia. 2011;66(3):191-212.

6. Sng BL, et al. Comparison of computer-integrated patient-controlled epidural analgesia and patient-controlled epidural analgesia with a basal infusion for labour and delivery. Anaesth Intensive Care. 2009;37(1):46-53.

7. Kandel PF, Spoerel WE, Kinch RA. Continuous epidural analgesia for labour and delivery: review of 1000 cases. Can Med Assoc J. 1966;95(19):947-53.

8. Bromage PR. Continuous lumbar epidural analgesia for obstetrics. Can Med Assoc J. 1961;85:1136-40.

9. Moore DC. Anesthetic techniques for obstetrical anesthesia and analgesia. Boston: Springfield; 1964.

10. Capogna G, Stirparo S. Techniques for the maintenance of epidural labor analgesia. Curr Opin Anaesthesiol. 2013;26(3):261-7.

11. Davies AO, Fettes IW. A simple safe method for continuous infusion epidural analgesia in obstetrics. Can Anaesth Soc J. 1981;28(5):484-7.

12. Boutros A, et al. Comparison of intermittent epidural bolus, continuous epidural infusion and patient controlled-epidural analgesia during labor. Int J Obstet Anesth. 1999;8(4):236-41.

13. Beilin Y, et al. Local anesthetics and mode of delivery: bupivacaine versus ropivacaine versus levobupivacaine. Anesth Analg. 2007;105(3):756-63.

14. COMETCSG UK. Effect of low-dose mobile versus traditional epidural techniques on mode of delivery: a randomised controlled trial. Lancet. 2001;358(9275):19-23.

15. Van der Vyver M, Halpern S, Joseph G. Patient-controlled epidural analgesia versus continuous infusion for labour analgesia: a meta-analysis. Br J Anaesth. 2002;89(3):459-65.

16. Halpern SH, Carvalho B. Patient-controlled epidural analgesia for labor. Anesth Analg. 2009;108(3):921-8.

17. Gambling DR, et al. A comparative study of patient controlled epidural analgesia (PCEA) and continuous infusion epidural analgesia (CIEA) during labour. Can J Anaesth. 1988;35(3 (Pt 1)): 249-54.

18. Paech MJ. Patient-controlled epidural analgesia in labour-is a continuous infusion of benefit? Anaesth Intensive Care. 1992;20 (1): 15-20.

19. Chua NP, Sia AT, Ocampo CE. Parturient-controlled epidural analgesia during labour: bupivacaine vs. ropivacaine. Anaesthesia. 2001;56(12):1169-73.

20. Ruban P, Sia AT, Chong JL. The effect of adding fentanyl to ropivacaine $0.125 \%$ on patient-controlled epidural analgesia during labour. Anaesth Intensive Care. 2000;28(5):517-21.

21. Sia AT, et al. Motor blockade is reduced with ropivacaine $0.125 \%$ for parturient-controlled epidural analgesia during labour. Can J Anaesth. 1999;46(11):1019-23.

22. Sia AT, Chong JL. Epidural $0.2 \%$ ropivacaine for labour analgesia: parturient-controlled or continuous infusion? Anaesth Intensive Care. 1999;27(2):154-8.

23. Chua SM, Sia AT. Automated intermittent epidural boluses improve analgesia induced by intrathecal fentanyl during labour. Can J Anaesth. 2004;51(6):581-5.

24. Fettes PD, et al. Intermittent vs continuous administration of epidural ropivacaine with fentanyl for analgesia during labour. $\mathrm{Br}$ J Anaesth. 2006;97(3):359-64.

25. Kaynar AM, Shankar KB. Epidural infusion: continuous or bolus? Anesth Analg. 1999;89(2):534.

26. Hogan Q. Distribution of solution in the epidural space: examination by cryomicrotome section. Reg Anesth Pain Med. 2002;27(2):150-6.

27. Lim Y, et al. Comparison of automated intermittent low volume bolus with continuous infusion for labour epidural analgesia. Anaesth Intensive Care. 2010;38(5):894-9. 
28. Sia AT, Lim Y, Ocampo C. A comparison of a basal infusion with automated mandatory boluses in parturient-controlled epidural analgesia during labor. Anesth Analg. 2007;104(3):673-8.

29. Leo $\mathrm{S}$, et al. A randomized comparison of automated intermittent mandatory boluses with a basal infusion in combination with patient-controlled epidural analgesia for labor and delivery. Int $\mathbf{J}$ Obstet Anesth. 2010;19(4):357-64.

30. Wong CA, et al. A randomized comparison of programmed intermittent epidural bolus with continuous epidural infusion for labor analgesia. Anesth Analg. 2006;102(3):904-9.

31. Wong CA, McCarthy RJ, Hewlett B. The effect of manipulation of the programmed intermittent bolus time interval and injection volume on total drug use for labor epidural analgesia: a randomized controlled trial. Anesth Analg. 2011;112(4): 904-11.

32. Capogna G, et al. Programmed intermittent epidural bolus versus continuous epidural infusion for labor analgesia: the effects on maternal motor function and labor outcome. A randomized double-blind study in nulliparous women. Anesth Analg. 2011;113(4):826-31.
33. - George RB, TK Allen, AS Habib. Intermittent epidural bolus compared with continuous epidural infusions for labor analgesia: a systematic review and meta-analysis. Anesth Analg. 2013; 116(1):133-44. First systematic review and meta-analysis comparing intermittent epidural bolus against continuous epidural infusions with or without PCEA.

34. Sia AT, Lim Y, Ocampo CE. Computer-integrated patient-controlled epidural analgesia: a preliminary study on a novel approach of providing pain relief in labour. Singapore Med J. 2006;47(11):951-6.

35. Lim Y, Sia AT, Ocampo CE. Comparison of computer integrated patient controlled epidural analgesia vs. conventional patient controlled epidural analgesia for pain relief in labour. Anaesthesia. 2006;61(4):339-44.

36. - Sia AT, S Leo, CE Ocampo. A randomised comparison of variable-frequency automated mandatory boluses with a basal infusion for patient-controlled epidural analgesia during labour and delivery. Anaesthesia. 2013;68(3):267-75. This study compared a new and unique concept of variable frequency automated boluses with basal infusion using new pump technology. 